\title{
La dependencia del Índice de Precios y Cotizaciones de la Bolsa Mexicana de Valores (IPC) con respecto a los principales índices bursátiles latinoamericanos
}

\author{
The dependence of the Price and Quotation Index of the Mexican Stock \\ Exchange (IPC) with respect to the main Latin American stock market indices
}

\author{
Roberto Joaquín Santillán Salgado ${ }^{1}$, César Gurrola Ríos²*, \\ Ana Lorena Jiménez Preciado ${ }^{3}$ y Francisco Venegas Martínez ${ }^{3}$
}

\author{
${ }^{1}$ Instituto Tecnológico y de Estudios Superiores de Monterrey, México \\ ${ }^{2}$ Universidad Juárez del Estado de Durango, México \\ ${ }^{3}$ Instituto Politécnico Nacional, México
}

Recibido el 06 de octubre del 2016; aceptado el 19 de enero del 2018

Disponible en Internet el 19 de septiembre de 2018

\begin{abstract}
Resumen
La intensidad y velocidad con la cual se transmiten los efectos de la política monetaria y fiscal de un mercado financiero a otro es de primordial importancia para calibrar con precisión las decisiones de las autoridades responsables. De manera similar, los efectos provocados por shocks inesperados en un mercado sobre el comportamiento de los precios de los activos financieros de otros mercados requieren una mejor comprensión de la naturaleza de la respuesta. Sin embargo, la mayoría de los estudios publicados sobre este tema han empleado metodologías que asumen normalidad en la distribución de los rendimientos y, por esa razón, sus resultados son cuestionables. Aunque en años recientes se han comenzado a utilizar metodologías robustas a la no-normalidad, aún falta mucho trabajo por realizar. Este artículo contribuye al estudio de la dependencia entre los índices de cuatro bolsas de valores latinoamericanas (el IPC de México, el IPSA de Chile, el IBOVESPA de Brasil, y el MERVAL de Argentina) mediante la metodología de Análisis de Cópulas. La principal contribución de este estudio con respecto a trabajos anteriores es la obtención del nivel de dependencia de las colas para los pares de índices formados por el IPC de México y cada uno de los otros tres índices latinoamericanos de la muestra.
\end{abstract}

Códigos JEL: C58; F65; G15.

Palabras clave: Mercados de capital latinoamericanos; cópulas bivariadas; relaciones de dependencia.

\footnotetext{
*Autor para correspondencia.

Correo electrónico: cgurrola@ujed.mx (C. Gurrola Ríos)

La revisión por pares es responsabilidad de la Universidad Nacional Autónoma de México.
} 


\begin{abstract}
The intensity and speed with which the effects of monetary and fiscal policy are transmitted from one financial market to another is of paramount importance to accurately calibrate the decisions of the responsible authorities. Similarly, the effects caused by unexpected shocks in a given market on the behavior of financial asset prices in other markets requires a better understanding of the nature of the response. However, the bulk of published studies on this subject have employed methodologies that assume a normal distribution of the yields and, for that reason, its results are questionable. Although in recent years robust non-normality methodologies have been used, there is still much work to be done. This article contributes to the study of the dependency between the indices of four Latin American stock exchanges (the CPI for Mexico, the IPSA for Chile, the IBOVESPA for Brazil, and the MERVAL for Argentina) through a Copula Analysis methodology. The main contribution of this study with respect to previous work is to obtain the level of dependence on the queues for the pairs of indices formed by the CPI of Mexico and each of the other three Latin American indices of the sample.
\end{abstract}

JEL classification: C58; F65; G15.

Keywords: Latin American capital markets; bivariate copulas; relations of dependence.

\title{
Introducción
}

En los últimos decenios se han observado correlaciones cruzadas cada vez más altas en los rendimientos de distintos mercados financieros, razón por la cual distintos autores han comenzado a estudiar el fenómeno y a documentar sus características (e.g., Licht, 1997; Forbes y Rigobon, 2002; Climent y Meneu, 2003; Aggarwal y Kyaw, 2005; Darrat y Zhong, 2005). El fondo de la discusión tiene que ver con la idea de que a medida que la globalización avanza y la integración financiera entre países y regiones se profundiza integración de portafolios diversificados se ha dificultado. Otra preocupación consiste en la observación de acontecimientos que provocan una volatilidad exacerbada en determinado mercado nacional, y se transmiten a otros mercados. En realidad, se trata de un mismo fenómeno, y la diferencia es solamente una cuestión de intensidad, pero en un caso se asocia con una tendencia gradual y, en el segundo, con una elevación súbita de la correlación entre los rendimientos de dos o más mercados nacionales.

La integración financiera global se ha visto propiciada por distintos factores como, por ejemplo, el fortalecimiento de acuerdos comerciales entre países y la decisión de los gobiernos de las principales potencias industriales de coordinar sus políticas económicas para moderar la volatilidad de los ciclos económicos, o bien, el "Efecto Fisher Internacional", en virtud del cual las fluctuaciones de la tasa de interés actúan como un mecanismo de atracción de flujos financieros entre país e se inciden sobre la valuación de los activos financieros (Choudhry, 1997) $\mathrm{o}$, incluso, la influencia favorable atribuible a la desregulación financiera, a la globalización de la economía y al avance tecnológico, porque contribuyen a una mayor eficiencia del mercado.

En su mayoría, los estudios de referencia a sumen una distribución normal de los rendimientos aunque existe amplia evidencia en contrario, ya que esa realidad es precisamente la que abre la puerta a la utilización de técnicas estadísticamente más robustas y capaces de capturar, además de las posibles relaciones lineales, también las relaciones no lineales. Tal es el caso de la metodología de cópulas, utilizada con creciente frecuencia para el estudio de fenómenos financieros, como puede constatarse en, por ejemplo, Calvo y Reinhart (1996), 
Chan-Lau, Mathieson y Yao, (2004), Bekaert et al. (2014), y Sosa, Bucio y Cabello (2015), por mencionar algunos de los publicados en años recientes.

Este trabajo examina empíricamente la estructura de las relaciones de dependencia en las colas pesadas de la distribución de los rendimientos de los índices de cuatro mercados bursátiles en América Latina mediante la técnica de cópulas bivariadas. La elección de la muestra de países estudiados respondió a una lógica muy sencilla: Argentina, Brasil, y México son las mayores economías de Latinoamérica, en tanto que Chile es probablemente el país con un mayor desarrollo relativo de su mercado de capitales con respecto al tamaño de su economía en la región.

Los rendimientos diarios de los índices bursátiles de los países de la muestra para el periodo del 21 de enero de 2005 al 20 de enero de 2016, para un total de 2,524 observaciones, se obtuvieron de Yahoo Finance. Las características de las series sugieren que las cópulas más apropiadas para modelar la dependencia son de tipo Joe-Clayton (BB7) y Survival (BB7), las cuales se caracterizan por tener diferentes valores de dependencia en la cola superior e inferior. Gracias a la metodología propuesta, es posible interpretar la interacción de los índices y las estructuras de orden superior presentes en los rendimientos, y concluir que las distribuciones marginales que mejor caracterizan a los índices bursátiles latinoamericanos se aproximan a una distribución logística similar a la normal, pero con colas más pesadas y curtosis más pronunciada; asimismo, que México y Brasil presentan las relaciones de dependencia más fuertes, tanto en la cola superior como en la cola inferior.

En la segunda parte de este trabajo se presenta una breve revisión de estudios sobre el tema de la transmisión de efectos entre mercados financieros, y se destacan los principales enfoques metodológicos y las herramientas más frecuentemente utilizadas. Finalmente, se mencionan algunos estudios que han utilizado la metodología de cópulas para resolver el problema de "no-normalidad" de los rendimientos financieros. En la tercera parte se presentan los aspectos metodológicos más relevantes de esta propuesta, así como los resultados obtenidos del análisis econométrico. Por último, la cuarta sección sintetiza los hallazgos y sugiere futuras líneas de investigación.

\section{Revisión de literatura}

La comprensión de los procesos subyacentes a la Globalización Financiera ha atraído el interés de numerosos investigadores. En este trabajo mencionamos solamente algunos de los más relevantes. En primera instancia, hacemos referencia a trabajos relacionados con el efecto de "contagio", cuya evidencia empírica se manifiesta cuando los precios de activos financieros cotizados en mercados de tamaño distinto, con estructuras disímbolas y ubicaciones geográficas diferentes reaccionan simultáneamente ante perturbaciones generadas, ya sea en alguno de ellos o, incluso, en mercados alejados y poco relacionados (Forbes y Rigobon, 2002; Gurgul y Machno, 2016). Un ejemplo es la crisis de las Hipotecas de Baja Calidad (Subprime) ${ }^{2}$ de $2007-$ 2012, considerada como la primera crisis global desde la Gran Depresión de los años 1930s, y que tuvo influencia significativa sobre el patrón de comportamiento de los rendimientos bursátiles de todo el planeta e incrementó los niveles de correlación entre mercados (por

\footnotetext{
${ }^{2}$ Para una revisión detallada de las crisis financieras revisar Calvo y Reinhardt (1996), Rigobon (2002), Mendoza, López y Watkins (2011) y Santillán (2015).
} 
ejemplo, Ffrench-Davis 2001; Aloui et al. 2013; Schwert 2011; Slimane et al.2013; Bekaert et al. (2014).

Ante la amplitud y profundidad de las crisis, el estudio de las formas cómo los acontecimientos que afectan a un mercado nacional se transmiten más allá de sus fronteras se ha vuelto uno de los temas de investigación de mayor interés en finanzas (Rigobon, 2002; Mollah y Hartman, 2012). El interés se justifica porque tales relaciones de dependencia afectan las decisiones de diversificación internacional, administración de riesgos, valuación de activos, y elaboración de portafolio (Boubaker y Sghaier, 2014).De manera puntual, Gurgul y Machno (2016) sostienen que el estudio de la transmisión de los efectos provocados por acontecimientos o fenómenos extraordinarios a través de las fronteras es importante no solamente para los inversionistas, sino también para los gobiernos. En el caso de éstos últimos, la transmisión de efectos a nivel internacional es relevante por la forma en que la política económica debe responder al impacto de las decisiones de inversión internacional sobre el tipo de cambio y la inflación. A su vez, los inversionistas y gestores de fondos también reaccionan a la información sobre el comportamiento conjunto de los precios, modifican sus decisiones de inversión o la oferta de sus productos.

\section{Principales enfoques sobre la transmisión de efectos financieros}

Algunos ejemplos de estudios sobre la propagación de efectos entre mercados financieros incluyen los trabajos de Calvo y Reinhart (1996), Rigobón (2002), Forbes y Rigobon (2002), Ning (2008), por mencionar solamente algunos.

Por ejemplo, según Calvo y Reinhart (1996) la mayoría de los estudios sobre el tema de contagio financiero pueden agruparse como: a) aquellos cuyo interés se concentra en el "comportamiento de rebaño" de los agentes económicos; y b) aquellos interesados en comprender los canales de transmisión del contagio. En éste último grupo se incluyen estudios que destacan el nivel de integración de los mercados de capital, acuerdos comerciales, prácticas institucionales, sesgo por mercados desarrollados, factores tecnológicos e inestabilidad política, como determinantes de la intensidad del contagio.

Rigobon (2002), asegura que la medición del contagio es una de las tareas más complicadas de las finanzas internacionales; analiza la definición clásica de contagio 3: "Contagion is the propagation of shocks among markets in excess of the transmission explained by fundamentals", y sostiene que es ambigua ${ }^{4}$, y que no puede ser probada. Argumenta que las propiedades estadísticas de las series financieras -heterocedasticidad, autocorrelación y ausencia de normalidad-impiden utilizar las herramientas tradicionales para capturar el contagio. Pero, aunque tales herramientas-coeficientes de correlación, componentes principales, regresiones lineales y probabilidades condicionales-, podrían ajustarse para resolver dichas limitaciones, la existencia de factores comunes no observables en la dinámica conjunta de los rendimientos complica el análisis. Él clasifica los esfuerzos por definir y capturar el contagio en dos grupos: a) "shift contagion"; y, b) "pure contagion". El primero refleja cambios en la intensidad de la transmisión de shocks, y abarca los cambios en los mecanismos de propagación durante una

\footnotetext{
${ }^{3}$ Para una amplia revisión sobre contagio remitirse a Calvo y Reinhart (1996), Rigobon (2002) y Mollah y Hartman (2012).

${ }^{4}$ Preguntándose aspectos como: ¿cuándo se consideran rendimientos en exceso? Y ¿qué entendemos por fundamentales?
} 
crisis financiera. En contraste, el "pure contagion" se refiere al mecanismo a través del cual se propagan los efectos. La clave está en identificar la proporción del shock que se transmite por los canales fundamentales; así, la parte no identificada constituirá el contagio propiamente dicho. La transmisión, más que una contingencia de corto plazo que responde a periodos de crisis focalizadas, es un proceso más o menos estable. Por lo tanto, el contagio se presenta sólo cuando la magnitud de la transmisión es inusualmente grande. Sobre la base de lo anterior se puede argumentar que las estrategias de política económica de corto plazo sólo sirven para retrasar el ajuste o recepción del shock externo.

A lo largo de los años 1990s, varios países emergentes experimentaron episodios de crisis y "contagio" no atribuibles a cambios en los fundamentales macroeconómicos hacia otras economías (Ffrench-Davis, 2001). Calvo y Reinhart (1996) encuentran que el contagio en mercados emergentes responde en mayor medida a factores regionales. Chan-Lau, Mathieson y Yao (2004) también documentan el efecto contagio y concluyen que éste es mayor cuando los mercados van a la baja (bear), que cuando hay rendimientos positivos (bull). Asimismo, presentan evidencia de que el contagio difiere en intensidad entre regiones; por ejemplo, los mercados de América Latina son más propensos a contagiarse, que lo que sucede con las economías de Asia.

En un trabajo de gran envergadura, Bekaert et al., (2014) estudian los efectos de la crisis subprime, y la manera como se transmitió rápidamente desde Estados Unidos hacia prácticamente todas las economías del mundo. Al analizar por medio de un modelo de factores el efecto de la crisis sobre el rendimiento de 415 portafolios integrados con activos financieros de distintos países, identifican correlaciones residuales y aumentos inexplicados en las cargas factoriales, las cuales confirman el contagio. Adicionalmente, el análisis revela que la crisis no se transmitió uniformemente, sino que tuvo mayor impacto en mercados con desequilibrios macroeconómicos y baja calificación crediticia.

No obstante, la abundante evidencia en validación de la existencia de contagios, algunos estudios señalan desventajas y limitaciones en su concepción o, incluso, como sugieren Lorenzo y Massa (2013), afirman que el contagio se confunde con las relaciones de dependencia. O bien, Rigobon (2002) donde al abordar las implicaciones teóricas y empíricas sobre el contagio, destaca algunos inconvenientes sobre su medición como, por ejemplo: a) las pruebas ignoran los efectos de la heterocedasticidad, por lo que son sesgadas y tienden a exagerar las interacciones normales entre los mercados; b) existen transmisiones posteriores a los shocks inesperados que son perfectamente normales y que, en ocasiones, se confunden con contagio; c) no obstante que el comercio explica casi la mitad de la transmisión de los shocks, una porción importante se debe a que los instrumentos financieros objeto del análisis cotizan simultáneamente en los mismos mercados de capital. Por su parte Uribe (2011) al señalar que el coeficiente de correlación de Pearson, modelos de vectores autorregresivos -VAR- y funciones de estímulorespuesta, suponen un comportamiento normal multivariado, supuesto poco realista en series financieras, asegura que carecen de utilidad inferencial.

\section{Contagio vs Dependencia}

En un análisis sobre la transmisión de efectos entre mercados financieros, Rigobon (2002) revisa algunas de las crisis financieras de los últimos decenios ${ }^{5}$, y comprueba que tuvieron

\footnotetext{
${ }^{5}$ Por ejemplo: la caída de la bolsa de Estados Unidos en 1987, la devaluación de peso mexicano en 1994, el desplome de la bolsa de Hong Kong en 1997, entre otras.
} 
eco en el comportamiento de los mercados financieros globales, lo cual puede interpretarse como una clara manifestación de contagio. No obstante, asegura, dicha interpretación puede ser errónea pues el contagio es más que la sola presencia de un elevado nivel de correlación entre mercados, aun cuando se vea incrementado después de un periodo de crisis en alguno de ellos. Al final de su estudio, Rigobon (2002) propone el término de "interdependencia" para definir tales relaciones.

$\mathrm{Hu}$ (2003) señala que la mayoría de los estudios sobre dependencia se ha orientado a capturar el "nivel" de dependencia, pero no se han preocupado por aislar la "estructura" de la misma. En su estudio, documenta que aun cuando los niveles de correlación son idénticos entre determinados mercados, es posible identificar diferentes estructuras de dependencia. Lorenzo y Massa (2013) analizan la dependencia entre mercados de capitales de economías emergentes latinoamericanas y coinciden con estudios previos en que tales estructuras no son estables. En particular, concluyen que dichas relaciones se han incrementado en el tiempo, especialmente después de la crisis subprime. Los autores sostienen que los rendimientos de activos financieros de las economías de América Latina presentan colas pesadas, es decir, tienen mayores probabilidades de presentar grandes pérdidas, y que los vínculos de dependencia se fortalecen en periodos de crisis, reduciendo los beneficios de la diversificación. Ning (2008) reconoce que durante periodos de crisis los vínculos entre los mercados se pueden volver más estrechos, y analiza las relaciones de dependencia en mercados accionarios internacionales. El análisis revela la presencia de dependencia asimétrica y relaciones directas con el estado de la economía. Asimismo, se reportan mayores niveles de dependencia en Europa y el Sudeste asiático, que en América.

Dos estudios recientes en los cuales se documentan diferencias regionales significativas en las relaciones dinámicas de dependencia entre mercados de capitales son los de Boubaker y Sghaier (2014) y Sosa, Bucio y Cabello (2015). En el primero de ellos se aborda el estudio de las relaciones de Estados Unidos, Japón, Reino Unido, Alemania y Francia, en tanto en el segundo se estudia la magnitud de las relaciones de dependencia entre los mercados de capitales pertenecientes al BRIC+M (Brasil, Rusia, India, China y México. Ambos estudios sugieren que los activos de los mercados de capital analizados ofrecen posibilidades de diversificación. Asimismo, en ambos casos se enfatiza que el enfoque más común para cuantificar el fenómeno de dependencia es utilizar el coeficiente de correlación de Pearson.

\section{Dificultades en la medición: coeficiente de correlación y falta de normalidad}

Una de las medidas más utilizada para evaluar la transmisión de efectos financieros es el coeficiente de correlación, pero varios autores sugieren que presenta limitaciones importantes. Por ejemplo, los trabajos de Forbes y Rigobon (2002), Hu (3003), Chan-Lau, Mathieson y Yao (2004), Lorenzo y Massa (2013), Sosa, Bucio y Cabello (2015) se han ocupado de profundizar en el análisis. Forbes y Rigobon (2002) señalan como debilidad importante del coeficiente de correlación su alta dependencia respecto a la volatilidad por lo que, durante los episodios de crisis, cuando ésta tiende a incrementarse, el parámetro tiende a sobreestimar la asociación entre mercados. Hu (3003) demuestra que el nivel de dependencia capturada en una distribución normal bivariada a partir de coeficientes de correlación, puede generar resultados que ofrezcan una idea errónea sobre las relaciones modeladas. Sosa, Bucio y Cabello (2015) también critican el uso de la correlación como medida de dependencia, aseguran que los parámetros 
estimados tienden a sobreestimarlas relaciones entre mercados, y destacan la incapacidad de las correlaciones para capturar relaciones no lineales y eventos extremos; en ello coinciden con Chan-Lau, Mathieson y Yao (2004) y Lorenzo y Massa (2013).

Otro aspecto a considerar es la heteroscedasticidad en el comportamiento de los rendimientos de las series financieras. Rigobon (2002), al estudiarla transmisión de efectos financieros, resalta la heterocedasticidad, autocorrelación y comportamiento no-normal de los rendimientos. $\mathrm{Hu}$ (2003) y Chan-Lau, Mathieson y Yao (2004) coinciden en que la modelación de distribuciones conjuntas para capturar las relaciones entre los rendimientos de activos intercambiados en distintos mercados, al asumir un comportamiento normal, produce resultados incorrectos. Ning (2008) critica los métodos que emplean correlaciones condicionales para capturar la dependencia, y coincide con Boubaker y Sghaier, (2014); Ortiz, Bucio y Cabello, (2016) y Oh y Patton, (2017).

\section{Ventajas de la metodología del análisis de cópulas}

Entre las alternativas propuestas para resolver las limitaciones metodológicas de los enfoques gausianos destacan dos conceptos estrechamente relacionados: las teorías de valores extremos ${ }^{6}$ y el análisis de cópulas; en este estudio se aborda únicamente el segundo de ellos. En particular, el análisis de cópulas representa una alternativa robusta para analizar las relaciones de dependencia. Para ello, se apoya en medidas de correlación no paramétricas como la $\rho$ de Spearman, y la $\tau$ de Kendall (Hu, 2003; Chan-Lau, Mathieson y Yao, 2004).

$\mathrm{Hu}(2003)$ afirma ser el pionero evidenciar las relaciones de dependencia entre mercados internacionales mediante un enfoque de cópulas mixtas, el cual favorece los patrones de la estructura de dependencia, pero no la intensidad de ésta. Su análisis revela que existen mayores posibilidades de "caídas" conjuntas versus comportamientos a la alza.

Alternativamente, Chan-Lau, Mathieson y Yao (2004) miden la dependencia con valores extremos y el uso de cópulas. Estos autores, concluyen que el efecto de contagio es mayor cuando los mercados van a la baja y presentan rendimientos negativos, que cuando hay rendimientos positivos; además, dicho efecto difiere de una región a otra.

Ning (2008) también propone la utilización de cópulas como una herramienta que permite capturar de una manera más robusta las relaciones de dependencia entre mercados financieros ante la presencia de valores extremos, y cuyo análisis revela la presencia de dependencia asimétrica en las colas. Alouiet al. (2013), Analizan las interdependencias extremas, y utilizan para ello cópulas que permiten analizar los patrones dinámicos de las colas pesadas y las interdependencias lineales y no lineales, y reportan dependencia variante en el tiempo entre los mercados BRIC y el mercado de Estados Unidos; vale la pena destacar que dichas relaciones son más intensas en aquellos mercados sensibles al precio de commodities, mientras que son menos intensas en economías exportadoras de productos manufacturados. Boubaker y Sghaier (2014), documentan diferencias regionales significativas en las relaciones dinámicas de dependencia entre los mercados de capitales de Estados Unidos, Japón, Reino Unido, Alemania y Francia, y para ello, utilizan la metodología de cópulas, generando las distribuciones marginales mediante la teoría de valores extremos, ajustando la dependencia en media y varianza condicionales. Un

\footnotetext{
${ }^{6}$ La teoría de valores extremos estudia la transmisión de efectos entre mercados financieros; dicha teoría se ha fortalecido ante la evidencia de que los mercados financieros reaccionan y transmiten, mediante distintos mecanismos, los shocks o crisis financieras, tomando en consideración que dicha transmisión es asimétrica, en función de la magnitud de las perturbaciones (ver, por ejemplo, Hu, 2003; Chan-Lau, Mathieson y Yao, 2004, Uribe y U1loa, 2012).
} 
estudio reciente, de Oh y Patton (2017), emplea nuevos modelos de cópula condicional variable en el tiempo para vincular las distribuciones marginales condicionales.

\section{Estudios en mercados emergentes}

Ortiz, Bucio y Cabello (2016) aseguran que la mayoría de los estudios sobre relaciones de dependencia entre mercados financieros publicados reportan evidencia empírica generada en economías desarrolladas, mientras que son pocos los estudios realizados en el contexto de mercados emergentes y menos aún, en mercados de América Latina.

Entre los trabajos publicados sobre las relaciones de dependencia en América Latina se incluye el de Christofi y Pericli (1999), quienes investigan la dinámica de corto plazo y analizan las distribuciones conjuntas con un modelo VAR con innovaciones que siguen un proceso GARCH exponencial. Los resultados de revelan dependencia entre los países estudiados así como mayores problemas de derrames de volatilidad (spillover) que en otras regiones del mundo. Otro trabajo que aborda la dependencia entre mercados de América Latina es el de Edwards y Susmel (2001), quienes responden la pregunta de si durante los períodos de alta volatilidad los mercados se correlacionan de manera diferenciada y centran su atención en los regímenes de codependencia de la volatilidad.El análisis revela volatilidad de estado-cambiante. En la misma línea, Arouri, et al. (2010) estudian las rupturas estructurales en la trayectoria de las correlaciones condicionales de los mercados de capital en la región, y encuentran que la intensidad de co-movimientos entre mercados ha cambiado a lo largo del tiempo, no obstante lo cual persisten las oportunidades de diversificación internacional de portafolios. Un último ejemplo de éste enfoque es el trabajo de Lorenzo y Massa (2013), quienes analizan el caso de los mercados de México y Brasil y proponen la cópula como una alternativa que considera la dependencia asintótica y que resuelve la falta de linealidad de la correlación simple. Además de hacer un llamado a no confundir la dependencia con el contagio, aseguran que los rendimientos de activos financieros de las economías de América Latina presentan colas pesadas.

Como exponente de una línea de investigación en la cual se abordan las relaciones de dependencia entre mercados de América Latina y mercados de otras regiones, el trabajo de Uribe (2011) estudia la incidencia del comportamiento del mercado de Estados Unidos sobre la economía colombiana. Mediante la utilización de cópulas y valores extremos su análisis revela que, en términos generales, la evidencia sobre contagio es incipiente, por lo que la inclusión de activos financieros del mercado colombiano puede contribuir a la diversificación de portafolios norteamericanos. Lo anterior puede explicarse, según el autor, como el resultado de la política económica de Colombia, que regula escrupulosamente el tránsito de capitales hacia el mercado doméstico. En el contexto del estudio del riesgo de crédito, Uribe y Ulloa (2012) también documentan la importancia de considerar la información contenida en las colas pesadas de las distribuciones a fin de mejorar los resultados ofrecidos por el VaR (Valor en Riesgo) tradicional, mediante la aplicación de la metodología GARCH y la estimación de parámetros mediante máxima verosimilitud. Dicho estudio compara los principales mercados de América Latina (Argentina, Brasil, México, Chile y Colombia) con algunos de economías desarrolladas y de otros mercados bursátiles emergentes. En el caso de Valenzuela y Rodríguez (2015), se analiza la existencia de interdependencias entre los seis principales mercados bursátiles de América Latina y los Estados Unidos, y se utiliza el estimador de Garman y Klass 
(1980) para analizar la volatilidad de los rendimientos bursátiles. Los resultados reportados por estos autores sugieren que existe una fuerte relación entre volatilidad y las correlaciones positivas entre los rendimientos de los mercados. Sosa, Bucio y Cabello (2015) estudian la magnitud de las relaciones de dependencia entre los mercados de capitales pertenecientes al BRIC+M (Brasil, Rusia, India, China y México), mediante cópulas y presentan evidencia sobre la existencia de niveles moderados de dependencia; es decir, sugieren que los activos de los mercados analizados ofrecen beneficios a la diversificación de portafolios. También, Ortiz, Bucio y Cabello (2016) analizan los rendimientos observados en economías del continente Americano: Canadá, Estados Unidos, México, Venezuela, Colombia, Perú, Brasil, Chile y Argentina, y estiman pérdidas potenciales, mediante la metodología de Value at Risk (VaR), a través de un enfoque de cópulas. El análisis permite concluir, entre otros aspectos, que las relaciones de dependencia son más fuertes en los países que integran el TLCAN, mientras que en el resto de las economías tal relación de dependencia es limitada por lo que la región, aseguran, ofrece interesantes posibilidades de diversificación. Finalmente, el trabajo de Bucio, De Jesús y Cabello (2016) reporta que un VaR soportado por análisis de cópulas captura de una manera más precisa los valores extremos típicamente observados en la dinámica de los activos financieros. Los autores resaltan la importancia de abordar el análisis de los rendimientos de los activos financieros respetando la naturaleza inherente a dicha información, es decir, considerando su comportamiento asimétrico y leptocúrtico.

Un hallazgo interesante de la revisión bibliográfica anterior es la confirmación la utilización cada vez más frecuente de la metodología de análisis de cópulas para modelar los patrones de dependencia entre variables financieras. Sin la necesidad de suposiciones previas sobre la distribución que siguen las series de rendimientos financieros, la teoría de cópulas permite incluir en la modelación de la dependencia los valores extremos observados y, utiliza las relaciones observadas en las colas pesadas (Hu, 2003; Chan-Lau, Mathieson y Yao, 2004; Kostadinov, 2005; Ning, 2008; Boubaker y Sghaier, 2014; Uribe y Ulloa, 2012; Ortiz, Bucio y Cabello, 2016; Bucio, De Jesús y Cabello, 2016).

\section{Metodología utilizada y resultados obtenidos}

Una copula asocia una función de distribución multivariada a sus distribuciones marginales uniformes, lo cual la convierte en una herramienta adecuada para modelar y simular variables aleatorias correlacionadas. El principal atractivo de las cópulas es que permiten capturar la estructura de dependencia y las distribuciones marginales por separado; por esta razón, se les llama también funciones de dependencia (Yan 2007).

$$
\left(u_{1}, \ldots, u_{p}\right)=P\left(U_{1} \leq u_{1}, \ldots, U_{p} \leq u_{p}\right)
$$

Puesto que las distribuciones marginales de las cópulas son en todos los casos uniformes, su distribución de probabilidad se encuentra en el intervalo $(0,1)$, y dado un vector aleatorio $U$ de p-dimensiones sobre el cubo unitario $[0,1]^{\mathrm{n}} x[0,1]$, la copula puede definirse como sigue:

De acuerdo con el Teorema de Sklar (1959), existe una cópula $C$ de p-dimensiones tal que para toda $x$ en el dominio de $F$ :

$$
F\left(x_{1}, \ldots, x_{p}\right)=C\left(F_{1}\left(x_{1}\right), \ldots F_{p}\left(x_{p}\right)\right)
$$


En el caso particular en el cual $F_{1}, \ldots, F_{p}$ son continuas, entonces $C$ es única; es decir, $C$ está unívocamente determinada por las distribuciones marginales. El Teorema de Sklar permite establecer la relación entre cópulas y funciones de distribución de variables aleatorias. Asimismo, no solo permite considerar a las cópulas como funciones de distribución conjuntas, sino que el argumento inverso también es cierto, por lo que a partir de las inversas de las distribuciones marginales $\left\{F_{1}^{-1}\left(x_{1}\right), \ldots F_{p}^{-1}\left(x_{p}\right)\right\}$ definidas en $[0,1]$, se obtienen las simulaciones de las variables aleatorias estudiadas.

Existe una amplia variedad de cópulas, pero las más utilizadas son las elípticas y las arquimedianas ${ }^{7}$. Sea $F$ la Función de Distribución Acumulada (FDA) de una distribución elíptica y sea $F_{i}$ la FDA de la i-ésima marginal y $F_{1}{ }^{-1}$ el cuantil (o función inversa), $i=1, \ldots, p$. La cópula elíptica determinada por $F$ está dada por:

$$
C\left(u_{1}, \ldots, u_{p}\right)=F\left(F_{1}^{-1}\left(u_{1}\right), \ldots, F_{p}^{-1}\left(u_{p}\right)\right)
$$

Entre las copulas elípticas, se incluye la cópula Gaussiana, la cual presenta una distribución normal, como sigue:

$$
C\left(u_{1}, \ldots, u_{p}\right)=F\left(\phi^{-1}\left(u_{1}\right), \ldots, \phi^{-1}\left(u_{p}\right)\right)
$$

Donde $F$ es la distribución normal conjunta y $\phi^{-1}$ es el cuantil de la distribución normal univariada. Asimismo, entre las cópulas elípticas está la t-Student, que presenta una distribución:

$$
C\left(u_{1}, \ldots, u_{p}\right)=t_{v, R}\left(t_{v}^{-1}\left(u_{1}\right), \ldots, t_{v}^{-1}\left(u_{p}\right)\right)
$$

Donde $t_{v, R}$ es la distribución conjunta t-Student y $t_{v}^{-1}$ es el cuantil de la distribución $t$-Student univariada con grados de libertad. A su vez, las cópulas arquimedianas, toman la forma:

$$
C_{\varphi}=\varphi\left(\varphi^{-1}\left(u_{1}\right)+\varphi^{-1}\left(u_{2}\right)+\cdots+\varphi^{-1}\left(u_{p}\right)\right)
$$

Donde $\varphi(t)$ es la función generadora y $\varphi^{-1}(t)$ es la inversa.A diferencia de las cópulas elípticas, las arquimedianas presentan amplios rangos de propiedades de dependencia a partir de la función generadora. Las más utilizadas son las conocidas como Clayton, Gumbel y Frank debido a que permiten modelar diferentes patrones de dependencia mediante formas funcionales simples. Dicho patrón de dependencia se denota por el parámetro , el cual representa el nivel o fuerza de dependencia.

En el caso particular de la presente investigación, se hace uso de cópulas bivariadas, por lo que se ajustan cópulas a los rendimientos de pares de mercados. En ese sentido, las tres principales cópulas arquimedianas más utilizadas presentan la forma:

\footnotetext{
${ }^{7}$ En el área de finanzas, las cópulas elípticas son muy populares debido a su fácil implementación, su función de distribución multivariada es simétrica además de presentar ventajas en obtener las distribuciones condicionales para su predicción (Sosa, Bucio, \& Cabello, 2015).
} 
Tabla 1

Familias de cópulas arquimedianas y función generadora.

\begin{tabular}{lccc}
\hline Familia & Función Cópula & Función Generadora & Parámetros \\
\hline Clayton & $\mathrm{C}_{\alpha}(\mathrm{u}, \mathrm{v})=\max \left(\left[\mathrm{u}^{-\alpha}+\mathrm{v}^{-\alpha}-1\right]^{-\frac{1}{\alpha}}, 0\right)$ & $\varphi_{\alpha}(\mathrm{t})=\frac{1}{\alpha}\left(\mathrm{t}^{-\alpha}-1\right)$ & $\alpha \geq 0$ \\
Frank & $\mathrm{C}_{\alpha}(\mathrm{u}, \mathrm{v})=-\frac{1}{\alpha} \ln \left[1+\frac{\left(\mathrm{e}^{-\alpha \mathrm{u}}-1\right)\left(\mathrm{e}^{-\alpha \mathrm{v}}-1\right)}{\mathrm{e}^{-\alpha}-1}\right]$ & $\varphi_{\alpha}(\mathrm{t})=-\ln \left(\frac{\exp (-\alpha \mathrm{t})-1}{\exp (-\alpha)-1}\right)$ & $\alpha \geq 0$ \\
Gumbel & $\mathrm{C}_{\alpha}(\mathrm{u}, \mathrm{v})=\exp \left\{-\left[(-\ln \mathrm{u})^{\alpha}+(-\operatorname{lnv})^{\alpha}\right]^{\frac{1}{\alpha}}\right\}$ & $\varphi_{\alpha}(\mathrm{t})=(-\ln \mathrm{t})^{\alpha}$ & $\alpha \geq 1$ \\
\end{tabular}

Fuente: Elaboración propia

Para los fines del presente estudio se estiman tres cópulas bivariadasque vinculan al IPC de México con cada una de las otras tres bolsas latinoamericanas (IBOVESPA, MERVAL e IPSA).

La Figura 1 muestra el comportamiento de los índices bursátiles de referencia, destacando que los datos fueron homogeneizados considerando sus días coincidentes de cotización. En lo que respecta al IPC y al IBOVESPA, se observa un comportamiento similar, por lo que a priori, se puede suponer alguna forma de correlación entre dichos índices. Sin embargo, esto no es fácilmente perceptible en el caso de los índices MERVAL e IPSA.

Figura 1. Comportamiento de los índices bursátiles latinoamericanos 21/01/2005 a 20/01/2016

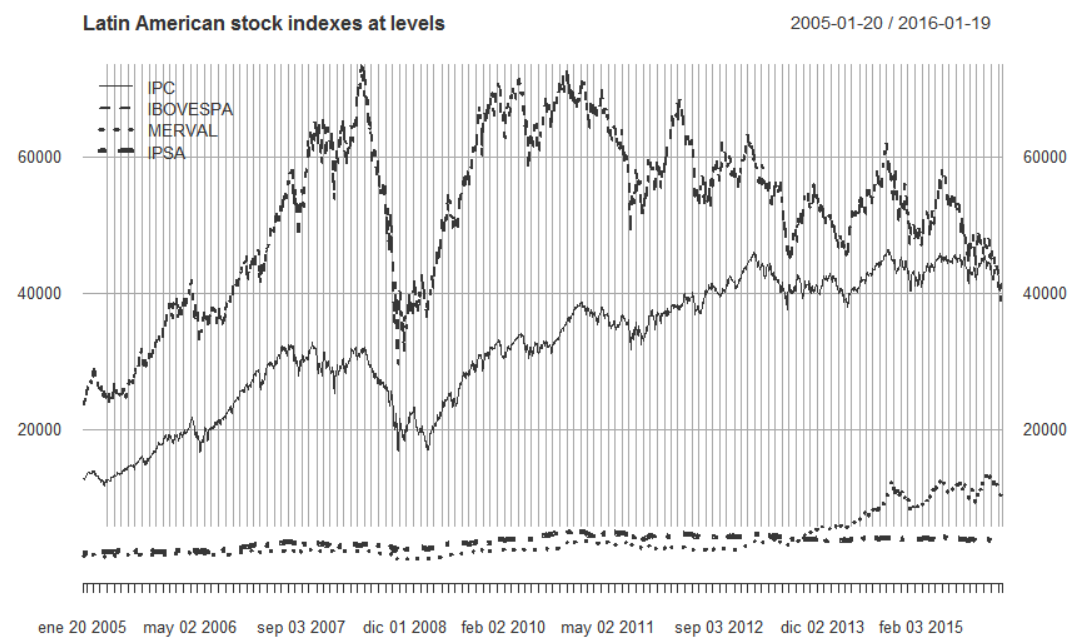

Fuente: Elaboración propia con base en Ryan J, Ulrich, J and Thielen, W. (2016): Quantitative Financial Modelling. $\mathrm{R}$ package versión $0.4-10$

La Figura 2 muestra los rendimientos de los índices de referencia durante el periodo analizado, y permite observar que los periodos de mayor volatilidad se presentaron durante el último trimestre del año 2008, como consecuencia de la crisis financiera originada en el sector inmobiliario de Estados Unidos. Es de llamar la atención que en el caso del IPSA, la 
turbulencia se observa durante un periodo más corto. Asimismo, en la Figura 2 se observa que el índice chileno es el que presenta menor volatilidad a lo largo de todo el periodo. Para México y Brasil, los rendimientos presentan mayor inestabilidad a finales de 2006 y durante 2008 y 2012 mientras que el MERVAL, además de los años mencionados, presenta un episodio de alta volatilidad en 2015, el cual no termina por mitigarse completamente aún en enero de 2016, y que se explica en alguna medida por los problemas políticos suscitados en ese país con el cambio de gobierno Kirschner-Macri.

Figura 2. Rendimiento de los índices bursátiles 21/01/2005 a 20/01/2016

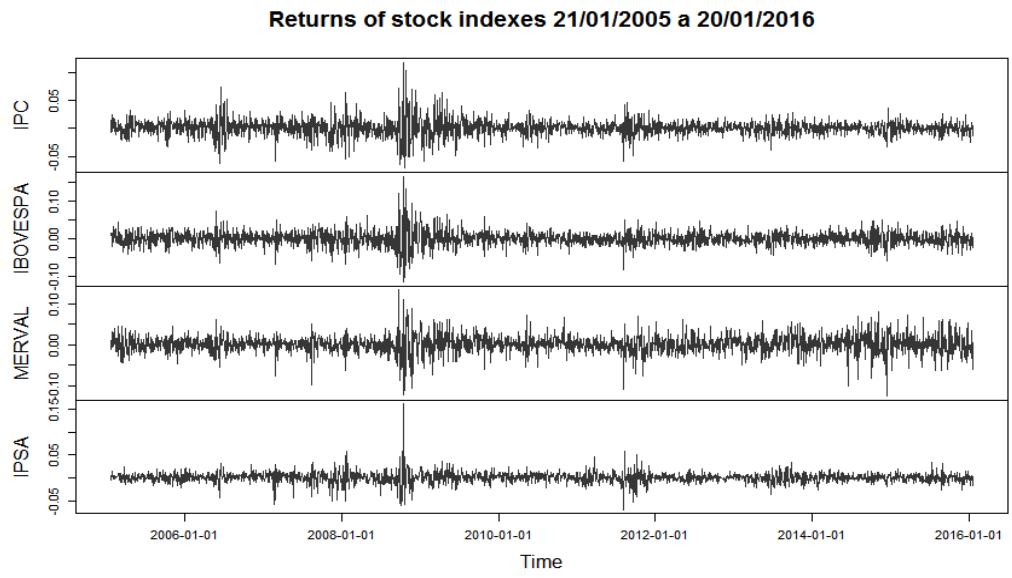

Fuente: Elaboración propia con base en Ryan J, Ulrich, J and Thielen, W. (2016): Quantitative Financial Modelling. $\mathrm{R}$ package versión $0.4-10$

La Figura 3 es una gráfica de cuantiles de las series de rendimientos y corrobora que ninguno de los índices se ajusta a una distribución normal. Este hecho resultaba evidente desde las figuras anteriores ya que, al presentarse clústers de volatilidad, existen problemas de heterocedasticidad en las series graficadas.

Para modelar la estructura de dependencia, en primera instancia, se utiliza una gráfica Chi-plot, mediante la cual se representa la dependencia local ${ }^{8}$ entre las variables. Propuesta por (Fisher \& Switzer, 1985),esta representación indica que si $y_{i}$ es una función estrictamente creciente de $x_{i}$, entonces $\chi=1$ y, en el caso opuesto, $\chi=-1$. A su vez, si las variables aleatorias son independientes, cuando $n \rightarrow \infty$, la distribución asintótica de $\lambda_{i}$ está uniformemente distribuida en un rango de $\pm 4\left(\left(\frac{1}{n}-1\right)-0.5\right)$.

\footnotetext{
${ }^{8}$ La dependencia local refiere a que ciertos subconjuntos de las variables aleatorias son independientes de los que están fuera de sus respectivas vecindades, por lo que no todos los valores registrados en las observaciones tienen influencia sobre otro.
} 
Figura 3 Gráfico Q-Q con distribución normal

Gráfico Q-Q IPC

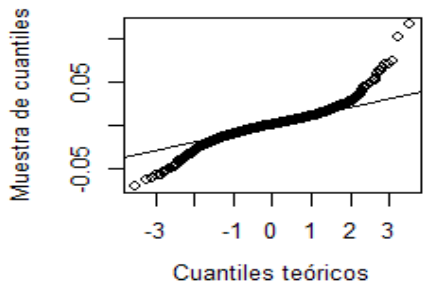

Gráfico Q-Q MERVAL

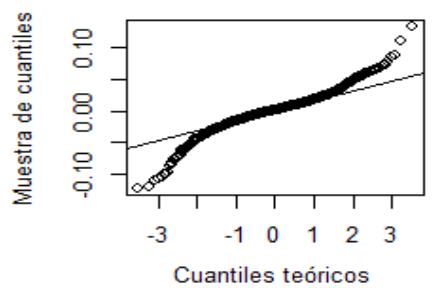

Gráfico Q-Q IBOVESPA

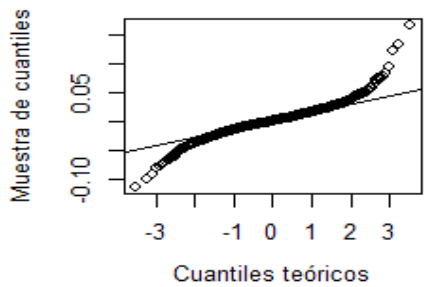

Gráfico Q-Q IPSA

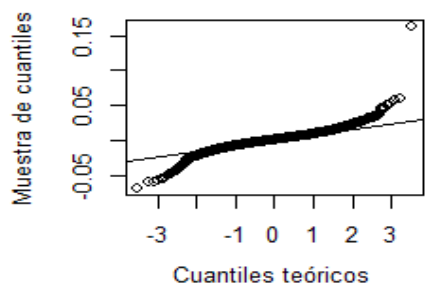

Fuente: Elaboración propia con base en R Core Team (2013): The R Stats. R package versión 3.3.0

Similar al caso de la correlación de Pearson, cuando existe independencia entre $y_{i}$ y $x_{i}$, entonces $\chi_{i}$ se distribuye aleatoriamente con un valor cercano a cero. La correlación positiva se da cuando $\lambda>0$ y, en el caso opuesto $\lambda<0$. La Figura 4 muestra los Chi-plots asociados a los índices bursátiles, tomando al IPC como variable de contraste. En los tres casos (IPCIBOVESPA, IPC-MERVAL e IPC-IPSA), se observa una dependencia negativa, aunque llama la atención la dispersión de los datos cuando $\lambda>0$ (correlación positiva), y las estructuras de dependencia positivas.

Figura 4 Chi-plot
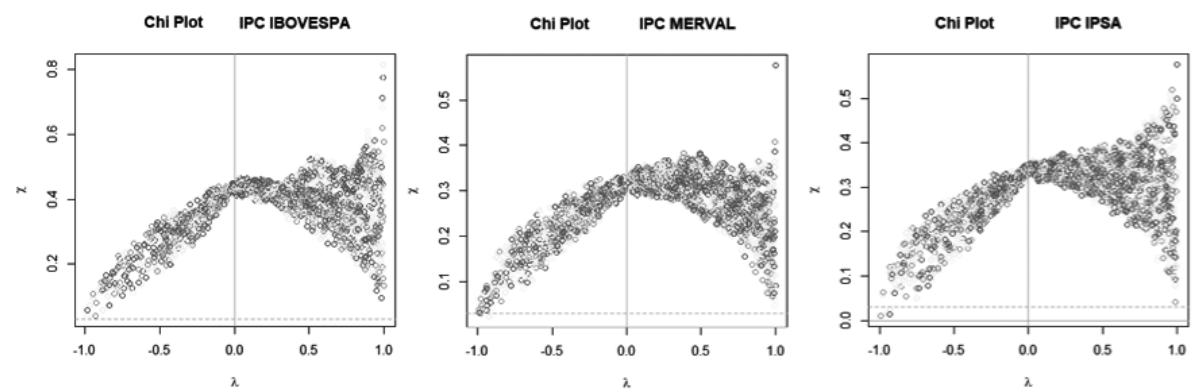

Fuente: Elaboración propia con base a Statistical Inference of Vine Copulas (2015): Schepsmeie. Ulf. R package versión 1.6-1

La Tabla 2, reporta la correlación entre el IPC y los demás índices bursátiles de la muestra con el coeficiente de correlación de Spearman y la Tau de Kendal. 
Tabla 2

Coeficientes de correlación entre el IPC y las distintas bolsas latinoamericanas: Tau de Kendal y Rho de Spearman

\begin{tabular}{|c|c|c|c|c|}
\hline \multirow[t]{2}{*}{ Índice bursátil/Prueba } & Tau de Kendall & Valor $\mathbf{p}$ & $\begin{array}{c}\text { Rho de } \\
\text { Spearman }\end{array}$ & Valor $\mathbf{p}$ \\
\hline & \multicolumn{2}{|c|}{ IPC } & \multicolumn{2}{|c|}{ IPC } \\
\hline IBOVESPA & 0.459 & 0.000 & 0.630 & 0.000 \\
\hline MERVAL & 0.346 & 0.000 & 0.489 & 0.000 \\
\hline IPSA & 0.355 & 0.000 & 0.500 & 0.000 \\
\hline
\end{tabular}

Fuente: Elaboración propia con base en McLeod, A. (2005): Kendall Rank correlation and Mann-Kendall trend test. R package versión 2.2

Aunque dichas medidas de correlación tienen diferentes valores para la misma muestra, si los test de significancia se basan en sus distribuciones de muestreo, se obtienen los mismos valores $p^{9}$ (Siegel y Castellan, 1988). Si bien ambas pruebas se utilizan para medir el nivel de correlación de datos no paramétricos entre dos variables, la más apropiada para muestras con colas correlacionadas es la Tau de Kendall (Gilpin, 1993), la cual se representa como sigue:

$$
\tau\left(x_{i}, x_{2}\right)=4 \iint_{0}^{1} C\left(u_{1}, u_{2}\right) d C\left(u_{1}, u_{2}\right)-1 ;
$$

es decir, se trata de una función de la cópula. Para la estimación de los parámetros correspondientes, se aplica una metodología de máxima verosimilitud. Retomando la Ecuación (2) se puede deducir que $C\left(x_{i}, x_{2}\right)=F\left(F_{1}^{-1}\left(x_{1}\right), F_{2}^{-1}\left(x_{2}\right)\right)$. Para estimar tanto los parámetros de las marginales como los parámetros de las cópulas (representados por $\theta$ ), se maximiza su función log-verosimilitud:

$$
L(\theta)=\sum_{i=1}^{n} \log c\left(F_{1}\left(x_{1 i}, x_{2 i}\right)\right)+\sum_{i=1}^{n} \sum_{i=1}^{2} \log f_{j}\left(x_{j i}\right)
$$

El estimador de máxima verosimilitud está dado por:

$$
\hat{\theta}=\max x_{\theta \in \theta} L(\theta)
$$

$\operatorname{con} \theta$, el espacio de los parámetros.

\section{Selección de distribuciones marginales}

Para seleccionar las distribuciones marginales, se hace uso de una gráfica de asimetríacurtosis propuesta por (Cullen \& Frey, 1999), basada en un re-muestreo o bootstrap para aproximar la distribución empírica de una muestra (en este caso, de cada uno de los índices bursátiles). Tanto los valores de asimetría como de curtosis correspondientes al re-muestreo están representados por un círculo en la Figura 5, como sigue:

\footnotetext{
${ }^{9}$ La hipótesis nula de ambas pruebas es que las variables no se encuentran correlacionadas.
} 
Figura 5 Gráfico de Cullen \& Frey para cada uno de los índices bursátiles
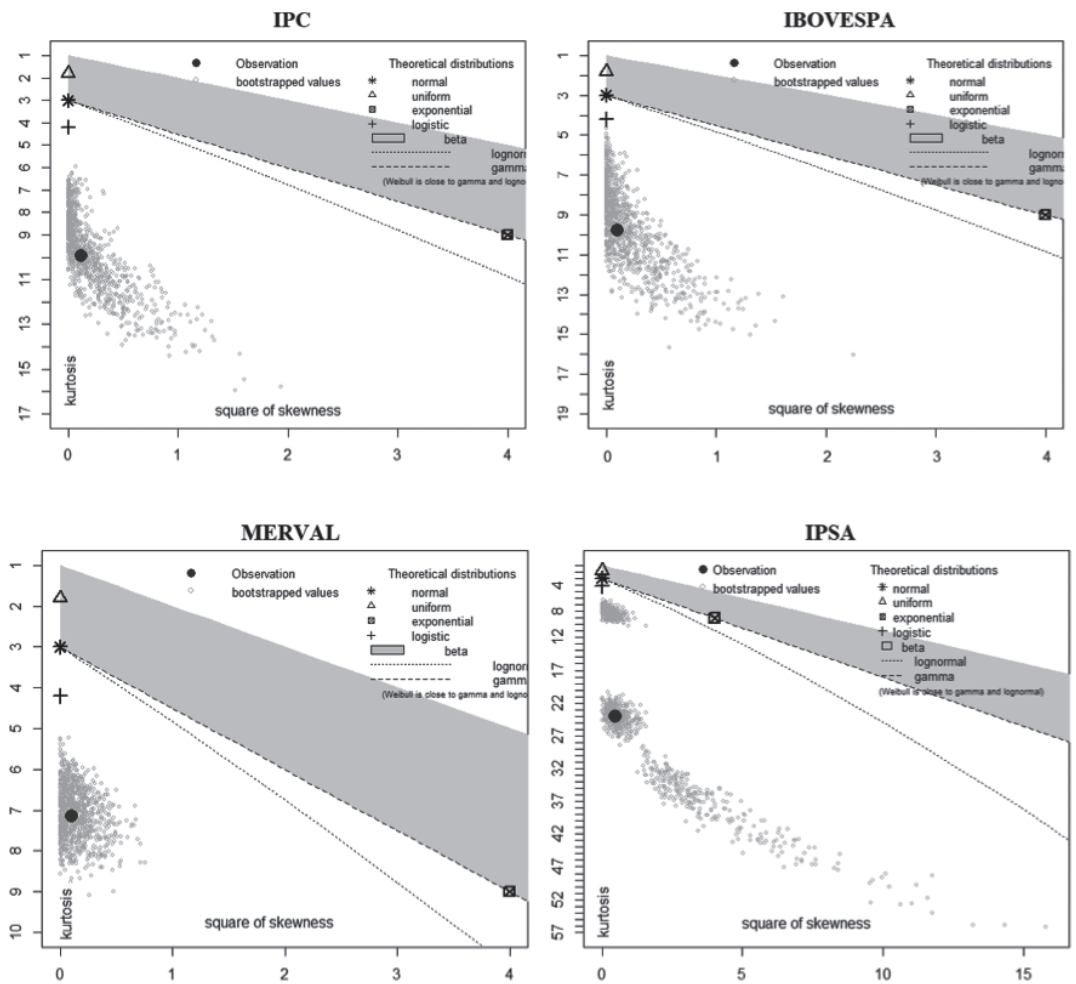

Fuente: Elaboración propia con base a Delignette-Muller et al. (2017): fitdistrplus: Help to Fit of a Parametric Distribution to Non-Censored or Censored Data. R package versión 1-0-9

La Figura 5 sugiere que, en todos los casos, las distribuciones marginales se aproximan más a una distribución logística. En este punto, vale la pena resaltar que la distribución logística es similar a la distribución normal, sólo que presenta colas más pesadas y curtosis más pronunciada, por lo que tiene la ventaja de ser más robusta, comparativamente. La función de densidad de una distribución logística está dada por:

$$
f(x ; \alpha, \beta)=\frac{e^{\frac{-(x-\alpha)}{\beta}}}{\beta\left(1+e^{\frac{-(x-\alpha)}{\beta}}\right)^{2}}
$$

Donde $\alpha$ es el parámetro de localización, $(-\infty \leq \alpha \leq \infty)$ y $\beta$ es el parámetro de escala $(0<\beta)$. Una vez obtenidas las distribuciones marginales, se hace uso de las pseudo observaciones de los indicadores bursátiles, es decir, las observaciones de las muestras transformadas sobre el intervalo $[0,1]$ con base en las marginales empíricas para discriminar entre las distintas familias 
de cópulas posibles, por medio de los criterios de Akaike (AIC) y el Bayesiano de Schwarz (BIC). Los resultados del proceso de selección se presentan en la siguiente tabla.

Tabla 3

Cópula bivariada seleccionada para los índices latinoamericanos

\begin{tabular}{cc}
\hline Relación de los índices & Cópula \\
\hline IPC, BVSP & Joe-Clayton (BB7) \\
IPC, MERV & Joe-Clayton (BB7) \\
IPC, IPSA & Survival Joe-Clayton (BB7) \\
\hline
\end{tabular}

Fuente: elaboración propia

De acuerdo con la Tabla 3, la familia que se ajusta mejor a la relación de dependencia entre el IPC-IBOVESPA y el IPC-MERVAL es la BB7 en tanto que para la relación IPCIPSA se obtuvo la misma cópula, pero rotada 180 grados (es decir, se trata de una cópula de supervivencia) cuyas propiedades se presentan en la Tabla 4.

Tabla 4

Propiedades de la cópula Joe-Clayton (BB7)

\begin{tabular}{c|c|c}
\hline Cópula & Función Generadora & Parámetros \\
\hline $\begin{array}{c}\text { BB7 y cópula de } \\
\text { supervivencia BB7 }\end{array}$ & $\left(1-(1-t)^{\theta}\right)^{-\delta}-1$ & $\theta \geq 1, \delta>0$ \\
\hline
\end{tabular}

Fuente: elaboración propia

$\theta$ y $\delta$ se refieren a los parámetros asociados a la cópula BB7, caracterizada por tener diferentes valores de dependencia en las colas superior e inferior. Quepa recordar que la familia Clayton no permite dependencia en la cola superior en tanto que la cópula Joe tiene la restricción de impedir dependencia en la cola inferior ${ }^{10}$. Por esa razón, el uso de la cópula BB7 permite modelar ambas estructuras de dependencia.

A partir de la utilización de la copula BB7 y de la cópula de supervivencia BB7, la Tabla 5 muestra los parámetros asociados a los índices bursátiles, el valor teórico la tau de Kendal para cópulas bivariadas, y los coeficientes de dependencia de las colas, dados los parámetros estimados.

La Tabla 5 presenta la correlación estimada con la cópula BB7 y las distribuciones marginales logísticas. Es importante destacar que se obtienen resultados similares a los presentados en la Tabla 2, lo cual sugiere que el ajuste de la cópula es adecuado para modelar la estructura de dependencia; asimismo, se presenta el nivel de dependencia de las colas para cada cópula. Por ejemplo, la relación IPC-BVSPA, la cópula en donde se observa mayor dependencia, depende

\footnotetext{
${ }^{10}$ Esto debido a su función generadora, por ejemplo, para la cópula Clayton $\psi(t)=(1+t), t \in[0, \infty]$ donde $\theta \in(0, \infty)$ En tanto que para la cópula Joe la función generadora es $1-(q-\exp (t))^{1 / \theta}$ donde $\theta \in(1, \infty)$.
} 
en $47 \%$ de la cola superior y $53 \%$ de la cola inferior. En la relación IPC-MERVAL se tiene una dependencia de la cola superior de $37 \%$, en tanto que en la cola superior de $31 \%$. Finalmente, la relación IPC-IPSA tiene una estructura de dependencia en la cola superior de $43 \%$ y la cola inferior de 29\%. Para los casos de Brasil y Argentina, la interacción tiene mayor efecto en la cola inferior, en tanto que la relación del IPC con el IPSA se concentra mayormente en los rendimientos positivos.

Tabla 5

Propiedades de la cópula Joe-Clayton (BB7)

\begin{tabular}{cccccc}
\hline $\begin{array}{c}\text { Relación de los } \\
\text { índices }\end{array}$ & $\begin{array}{c}\text { Parámetro } \\
\mathbf{1}\end{array}$ & Parámetro 2 & $\begin{array}{c}\text { Tau } \\
\text { Kendall }\end{array}$ & Cola superior & Cola inferior \\
\hline IPC, BVSP & 1.6347 & 1.0906 & 0.4615 & 0.4719 & 0.5296 \\
IPC, MERV & 1.3250 & 0.7002 & 0.3430 & 0.3127 & 0.3716 \\
IPC, IPSA & 1.5407 & 0.5682 & 0.3646 & 0.4318 & 0.2953 \\
\hline
\end{tabular}

Fuente: elaboración propia

\section{Conclusiones}

La transmisión de información entre mercados financieros contribuye a la formación de precios por lo que los avances en la tecnología de información y cómputo, la desregulación financiera y la cada vez mayor transparencia con la que se maneja la información financiera, son factores a favor. Pero adicionalmente a los factores puramente financieros, existen elementos de comportamiento de los mercados difíciles de clasificar, pero que se manifiestan en la manera cómo reaccionan ante la profundización de las relaciones comerciales y contractuales entre mercados nacionales, pero también, de manera especialmente intensa, como respuesta a acontecimientos de pronóstico difícil y, en ocasiones, visiblemente peligrosos para la estabilidad. La literatura sobre la creciente integración financiera de largo plazo entre los países se complementa con aquellos estudios que proponen mostrar los fenómenos de contagio producidos como respuesta a eventos inesperados para configurar un cuerpo de conocimiento creciente y de la mayor relevancia para las autoridades financieras y monetarias, pero también especialmente relevante para los administradores de portafolios y los inversionistas en general. Cabe mencionar que la incursión en este tema es aún incipiente y la variedad de estudios publicados difícilmente coinciden en sus conclusiones, por lo que es de esperar un desarrollo notable en la investigación sobre esta temática en los próximos años.

El presente estudio utiliza el análisis de cópulas para estudiar las relaciones de dependencia entre el IPC del mercado de valore mexicano con respecto a los mercados de Argentina, Brasil y Chile. El enfoque es innovador desde el punto de vista metodológico, pero también constituye una contribución interesante para el mejor conocimiento de la dinámica de los mercados financieros de la región. 
Una diferencia importante con respecto a otros trabajos que abordan el tema es la obtención del nivel de dependencia de las colas para los pares de índices formados por el IPC de México y cada uno de los otros tres índices latinoamericanos. Los hallazgos más importantes que se presentan son los siguientes:

- La relación entre el IPC y el IBOVESPA presenta la dependencia más fuerte, tanto en la cola superior como en la cola inferior ( $52.96 \%$ y $46.15 \%$ respectivamente).

- Las distribuciones marginales que mejor caracterizan a los índices bursátiles latinoamericanos se aproximan a una distribución logística, similar a la normal, pero con colas más pesadas y curtosis más pronunciada.

- Las familias de cópulas bivariadas que mejor caracterizan la distribución conjunta de los índices son las arquimedianas. Específicamente, la Cópula Joe-Clayton BB7, permite modelar ambas estructuras de dependencia con buena precisión.

Conocer mejor las relaciones de dependencia entre los mercados bursátiles latinoamericanos contribuye a mejorar la toma de decisiones de inversión y financiamiento de las empresas de la región, de los administradores de portafolios y de los inversionistas en general. También puede aportar información valiosa para el diseño de políticas públicas, esquemas regulatorios y autoridades supervisoras. La diversidad de temas asociados a la dependencia y, en casos excepcionales, el contagio entre mercados, representa un área fértil y de gran interés para futuras investigaciones.

\section{Referencias}

Aggarwal, R., \& Kyaw, A.N (2005). Equity Market Integration in the NAFTA Region: Evidence from Unit Root and Cointegration Tests.International Review of Financial Analysis. 14(4): 393-406. https://doi.org/10.1016/j. irfa.2004.10.008

Aloui, R., M. S., Aïssa, Ben \& Nguyen, D. K. (2013). Conditional Dependence Structure between Oil Prices and Exchange Rates: A Copula-GARCH Approach. Journal of International Money and Finance. 32(2), 719-738. https:// doi.org/10.1016/j.jimonfin.2012.06.006

Arouri, M. E. H., Bellalah, M., \& Nguyen, D. K. (2010). The Comovements in International Stock Markets: New Evidence from Latin American Emerging Countries. Applied Economics, 17(13): 1323-1328. https://doi. org/10.1080/13504850902967449

Bekaert, G., Ehrmann, M., Fratzscher, M., \& Mehl, A. (2014). The global crisis and equity market contagion. The Journal of Finance, 69(6), 2597-2649. https://doi.org/10.1111/jofi.12203

Boubaker, H., \& Sghaier, N. (2014). On the dynamic dependence between US and other developed stock markets: An extreme-value time-varying copula approach. Ipag Business School Working PaperNo. 2014-094. Disponible en: http://www.ipagcn.com/wp-ontent/uploads/recherche/WP/IPAG_WP_2014_281.pdf ; consultado el 21/08/2018

Bucio, C., De Jesús, R., \& Cabello, A. (2016). Valor en riesgo anual de los mercados accionarios de México y Estados Unidos: VaR tradicional vs VaR cópulas elípticas. Estocástica: finanzas y riesgo, 6(1), 83-114. Disponible en: http://estocastica.azc.uam.mx/index.php/re/article/viewFile/38/35, consultado el 21/08/2018

Calvo, S., \& Reinhardt, C. (1996). Capital flows to Latin America: Is There Evidence of Contagion Effects?Policy Research Working Paper 1619.The World Bank, International Monetary Fund. https://doi.org/10.1596/1813-94501619

Christofi, A., \& Pericli, A. (1999). Correlation in Price Changes and Volatility of Major Latin American Stock Markets. Journal of Multinational Financial Management, 9(1): 79-93. https://doi.org/10.1016/s1042-444x(98)00047-4

Chan-Lau, J. A., Mathieson, D. J., \& Yao, J. Y. (2004). Extreme contagion in equity markets. IMF staff papers, 51(2), 386-408. https://doi.org/10.2139/ssrn.382502 
Climent, F., \& Meneu, V. (2003). Has 1997 Asian Crisis Increased Information Flows between International Markets?International Review of Economics and Finance, 12(1): 111-143. https://doi.org/10.1016/s1059-0560(02)00140-5

Choudry, T. (1997). Stochastic Trends in Stock Prices: Evidence from Latin American Markets.Journal of Macroeconomics, 19(2): 285-304. https://doi.org/10.1016/s0164-0704(97)00016-5

Cullen, A.C. and H.C. Frey, H.C. (1999). Probabilistic Techniques in Exposure Assessment, Plenum Publishing Corp., New York, USA

Darrat, A. F., \& Zhong, M. (2005). Equity Market Linkage and Multinational Trade Accords: The Case of NAFTA. Journal of International Money and Finance, 24(5): 793-817. https://doi.org/10.1016/j.jimonfin.2005.04.006

Edwards, S., \& Susmel, R. (2001). Volatility Dependence and Contagion in Emerging Equity Markets. Journal of Development Economics, 66(2): 505-532. https://doi.org/10.1016/s0304-3878(01)00172-9

Ffrench-Davis, R. (2001). Crisis financieras en países exitosos. CEPAL. McGraw-Hill Interamericana.

Fisher, N., \& Switzer, P. (1985). Chi-plots for assessing dependence. Biometrika, 72, 253-265. https://doi.org/10.1093/ biomet/72.2.253

Forbes, K. J., \& Rigobon, R. (2002). No Contagion, Only Interdependence: Measuring Stock Market Co-movements? Journal of Finance, 57(5): 2223-2261. https://doi.org/10.1111/0022-1082.00494

Garman, M. B., \& Klass, M. J. (1980). On the Estimation of Security Price Volatilities from Historical Data. The Journal of Business, Vol. 53, No. 1 (Jan. 1980), pp. 67-78. https://doi.org/10.1086/296072

Gilpin, A. R. (1993). Table for conversion of Kendall's Tau to Spearman's Rho within the context of measures of magnitude of effect for meta-analysis. Educational and Psychological Measurement, 53(1), 87-92. http://dx.doi. org/10.1177/0013164493053001007

Gurgul, H., \& Machno, A. (2016). Modeling dependence structure among European markets and among Asian-Pacific markets: a regime switching regular vine copula approach. Central European Journal of Operations Research, 24(3), 763-786. https://doi.org/10.1007/s10100-015-0411-x

Hu, L. (2003). Dependence Patterns Across Financial Markets: Methods and Evidence. Mimeo. Department of Economics. Ohio State University. USA.

Kostadinov, K. (2005). Non-parametric estimation of elliptical copula with application to credit risk. Research paper, Munich University of Technology. Disponible en: http://citeseerx.ist.psu.edu/viewdoc/download?doi=10.1.1.61.3953\&rep=rep1\&type=pdf; consultado el 21/08/2018

Licht, A. N. (1997). Stock Market Integration in Europe. Program on International Financial Systems, Harvard Law School. Disponible en: http://pdf.usaid.gov/pdf_docs/PNACH173.pdf; consultado el 21/08/2018

Lorenzo, Valdés A., \& Massa, Roldán, R. (2013). Measuring Dependence in Financial Crisis A Copula Approach for Mexico and Brazil. Economía Mexicana. Nueva Época, 22(2) 341-355. Disponible en: http://www.economiamexicana.cide.edu/indice_articulos.html; consultado el 21/08/2018

Mendoza, Velázquez A., López, Herrera F. y Watkins, Fassler K. (2011), Reflexiones sobre las crisis financieras, Centro de Investigación e Inteligencia Económica CIIE-UPAEP y División de Investigación de la Facultad de Contaduría y Administración, UNAM. Coordinadores.

Mollah, S., \& Hartman, T. (2012). Stock Market Contagion, Interdependence and Shifts in Relationship due to Financial Crisis-A Survey. International Review of Business Research Papers, 8(1), 166-195. Disponible en: https:// www.bizresearchpapers.com/10.\%20Sabur.pdf; consultado el 21/08/2018

Ning, C. (2008). Extreme dependence of international stock market. Working paper, Ryerson University. Disponible en: http://digital.library.ryerson.ca/islandora/object/RULA:182/datastream/OBJ/download/Extreme_Dependence_in_International_Stock_Markets.pdf, consultado el 21/08/2018

Oh, D. H., \& Patton, A. J. (2017). Time-varying systemic risk: Evidence from a dynamic copula model of CDs spreads. Journal of Business \& Economic Statistics, 1-15. https://doi.org/10.1080/07350015.2016.1177535

Ortiz, E., Bucio, C., \& Cabello, A. (2016). Dependence and Value at Risk in the Stock Markets from the Americas: A Copula Approach. Journal of Research in Business, Economics and Management, 5(5), 761-780. Disponible en: http://www.scitecresearch.com/journals/index.php/jrbem/article/view/734; consultado el 21/08/2018

Rigobon, Roberto (2002). International Financial Contagion: Theory and Evidence in Evolution, CFA. The Research Foundation Publications. Disponible en: https://www.cfainstitute.org/en/research/ foundation/2002/international-financial-contagion-theory-and-evidence-in-evolution; consultado el 21/08/2018 
Santillán Salgado, R.J. (2015). La Gran Recesión (2007-2012): Lecciones y Oportunidades para México. EGADE Business School-Instituto Mexicano de Ejecutivos de Finanzas, PWC. Roberto J. Santillán-Salgado, Coordinador.

Sosa, M., Bucio, C., \& Cabello, A. (2015). Mercados de Capitales del Bloque Bric+ México: Dependencia Estimada con un Enfoque de Cópulas. Investigación Administrativa, 44 (115), 70-86. Disponible en: http://www.sepi.escasto.ipn.mx/Revista/Paginas/articulos115.aspx; consultado el 21/08/

Schwert, G. W. (2011). Stock Volatility during the Recent Financial Crisis. European FinancialManagement,17(5):789-805.https://doi.org/10.1111/j.1468-036x.2011.00620.x

Siegel, S., \& Castellan, N. J. (1988). Nonparametric statistics for the behavioral sciences (2nd ed.) New York: McGraw-Hill.

Sklar, A. (1959). Fonctions de répartition à n dimensions et leurs marges, Vol. 8, pp. 229-231, Publications de l’Institut de Statistique de L'Université de Paris.

Slimane, F., Mehanaoui, M. \& Kazi, I. (2013). How Does the Financial Crisis Affect Volatility Behavior and Transmission Among European Stock Markets? International Journal of FinancialStudies, 1(3): 81-101. https://doi. org/10.3390/ijfs 1030081

Uribe, J. (2011). Contagio financiero: una metodología para su evaluación mediante coeficientes de dependencia asintótica. Lecturas de Economía, (75), 29-57. Disponible en: http://www.redalyc.org/pdf/1552/155222750002.pdf, consultado el 21/08/2018

Uribe, J. \& Ulloa, I. (2012). La medición del riesgo en eventos extremos. Una revisión metodológica en contexto. Lecturas de Economía, No. 76, 87-117. Disponible en: http://www.scielo.org.co/scielo.php?pi$\mathrm{d}=$ S0120-25962012000100004\&script=sci_abstract\&tlng=fr; consultado el 21/08/2018.

Valenzuela, G. \& Rodríguez, A. (2015). Interdependencia de mercados y transmisión de volatilidad en América Latina. Innovar, 25(55): 157-170. https://doi.org/10.15446/innovar.v25n55.47231

Yan, Jun (2007). Enjoy the Joy of Copulas: With a Package copula. Journal of Statistical Software. October 2007, Volume 21, Issue 4. Disponible en: http://citeseerx.ist.psu.edu/viewdoc/download?doi=10.1.1.610.7783\&rep=rep1\&type=pdf; consultado el 21/08/2018 